\title{
The Construction and Analysis of the Media Image of the Elderly in Qilu Evening News
}

\author{
Huanning Yan ${ }^{1}$, a, Weifeng $\mathrm{Li}^{2}$, b \\ ${ }^{1}$ School Of Political Science And Law University Of Jinan, Shandong 250022, China; \\ ${ }^{2}$ School Of Political Science And Law University Of Jinan, Shandong 250022, China. \\ a1170107233@qq.com, bliweifeng96@163.com
}

Keywords: Qilu Evening News, Reports of the elderly, Media image.

\begin{abstract}
With the increasing number of elderly people, the elderly population has become one of the main audiences of the mass media, and it is very important to build an image of the elderly in the mass media. Here we will discuss about Qilu Evening News as a sample, through the content analysis method, it analyzes the reports of the elderly in the period from 2001 to 2010, and studies the image of the elderly in the Qilu Evening News. What are the trends, what factors are affected, and recommendations for active elderly reports.
\end{abstract}

\section{Introduction}

Since China entered an aging society in 2000, the number of elderly people in China has continued to surge. At present, China has become the country with the largest number of elderly people in the world. ${ }^{[1]}$ According to relevant experts, by 2050 , China's elderly people aged 60 and over will reach one-fifth of China's total population and enter the ranks of ultra-aging countries. ${ }^{[2]}$ With the continuous increase of the elderly population, the proportion of the elderly audience is also rising. In theory, the elderly will become one of the main audiences of the mass media, so the research on the image of the elderly built in the mass media will be changed. It is very important. ${ }^{[3]}$

In a general sense, content analysis is a research method of social science, ${ }^{[4]}$ here, using the definition of Berelson, he believes: "Content analysis is an objective of the dissemination of content with clear characteristics systematic and quantitatively described research techniques." [5]

The Qilu Evening News is a provincial-level comprehensive daily newspaper. It is the largest circulation newspaper in Shandong Province with the highest advertising revenue and the strongest social influence. ${ }^{[6]}$ Selecting the report of the elderly in the Qilu Evening News as the research object, and having a certain representativeness on the study of the image of the elderly in the newspapers in China.

Through the content analysis method, the elderly report from 2001 to 2010 was analyzed. According to the principle of "ten every ten draws one", the sample content was scrolled and a total of 360 days of news reports were taken as samples for three days. In less than 30 days in February, the last day is taken as a sample.

At the same time, the author refers to the classification of the previous scholars and Wang Shifan's "Communication Content Analysis Methodology and Evidence"and the analysis categories designed by the predecessors, comprehensively the purpose and needs of the actual research, and determine the specific sample selection principles and contents. Analyze the category, describe the image of the elderly from the overall and specific aspects, the specific image of the elderly includes the characteristics of the object of the report, the subject of the report and the position of the report.

\section{2. the characteristics of the image of the elderly in Qilu Evening News}

\subsection{Insufficient share of the image of the elderly}

A sample of the Qilu Evening News from 2000 to 2010 was selected, and a total of 38,544 news was collected in 360 days. The total number of reports of the elderly was 506, accounting for 
$1.313 \%$, which proved that the elderly reported a very low proportion One percent. The results of the sixth census show that the number of elderly people aged 60 and over in China has reached 178 million, accounting for $13.26 \%$ of the total population. ${ }^{[7]}$ It can be seen that the report of the elderly is much lower than that of the elderly in the society.

Hu Ling once mentioned: "The bias of the number of reports implies a reporter's stereotype of the importance of people or things, social status, and social roles". ${ }^{[8]}$ The low proportion of reports of the elderly reflects the low status of the elderly. The Qilu Evening News did not pay much attention to this group. Zhang Ronghua, Yuan Bingbing and other scholars have also proved this point, and this conclusion is consistent with previous studies.

\subsection{The imbalance of the gender ratio and urban-rural ratio}

In the 506 elderly report, only 205 news articles were reported for male elderly people, accounting for $40.51 \%$; only 140 news reports of female elderly were reported, accounting for $27.67 \%$. The proportion of female elderly reports is lower than that reported by males, which is about seven tenths. In the 506 reports of the elderly, there were 182 reports on urban elderly, accounting for $35.97 \%$; only 93 reports on rural elderly, accounting for $18.38 \%$, accounting for about two-thirds of urban elderly reports.

The content expressed by the mass media is limited and cannot reflect all aspects of society. Therefore, its content is "selected". Affected by Chinese traditional culture and social reality, the proportion of reports on older women is much lower than that of men in older people's reports. The attention of urban elderly is much higher than that of rural elderly.Ren Chunxiang and Qi Jiyuan also put forward this point in their research. The author's conclusion is consistent with previous studies.

\subsection{The inconsistent of the physiological and psychological images}

In the image report of the elderly, there are 48 reports of healthy physiological images, accounting for $9.49 \% ; 137$ cases of unhealthy physiological images, accounting for $27.08 \%$, which is about the physiological image of healthy elderly people. 3 times. At the same time, in the psychological image report of the elderly, there are 197 news reports on the positive image of the elderly, accounting for $38.93 \%$; there are 63 reports on the negative psychological image of the elderly, accounting for $12.45 \%$. In the shaping of its social image, there are 200 news reports on the positive social image of the elderly, accounting for $39.53 \%$; there are 129 reports on the negative social image of the elderly, accounting for $25.50 \%$. Among the negative elderly image reports, there are 54 reports of elderly people who need help, accounting for $41.86 \%$ of negative image reports.

It can be seen that the physiological image of the elderly is mainly unhealthy. The elderly often appear as the physiological image of "aging and useless" and "frail and sick", while in the psychological image of the elderly, they are mostly positive and positive. The physiological image displayed by the elderly is inconsistent with the inner psychological image and presented in a contradictory state. In the course of his research, Xu Jin and Zhu Jinping scholars also put forward the view that the image of the elderly is mostly negative and the image of the elderly has discriminatory bias. The author's conclusion is similar to previous studies.

\subsection{The neutral and positive image of the elderly}

According to the specific "news framework", the media will select the parts or aspects that they think are "important" from the real environment, and give them a certain structural order, and then present them to the audience. Therefore, the reporting position plays a crucial role in shaping the image of the elderly. In the 506 reports, there were 325 elderly people with a neutral attitude, accounting for $64.23 \%$; 151 with positive attitudes, accounting for $29.84 \%$; and 30 with negative attitudes. The proportion is $5.93 \%$. The proportion of affirmative and neutral positions in older people's reports is much higher than the proportion of negative positions.

It can be seen that in the report of the elderly, the staff members have a neutral and affirmative stance, and the image of the elderly is relatively impressive, which is slightly different from 
previous studies. The reason is closely related to the respect for the old culture in Shandong Province. Respecting the old and loving the young is the traditional virtue of our country, especially in Shandong, the hometown of Confucius and Mencius. In today's increasingly aging society, tapping and promoting the traditional culture of respecting the elderly and respecting the elderly is of great significance for caring for the elderly and building a harmonious society.The Qilu Evening News is also related to the traditional culture in shaping and reporting the image of the elderly.

\section{Analysis of factors affecting the image of the elderly}

\subsection{The influence of social and cultural factors}

The content of communication in the mass media is not isolated. It interacts with cultural environments such as inherent cultural traditions and values. Hu Ling once mentioned: "The history and culture, traditional norms and values of a society will often create a common mindset for its members of society and become a fixed pattern for people to think about the objective world."[9] The presentation of the content of the communication reflects certain Cultural traditions.

First of all, the traditional thinking of "male and female" and "male and weak" has become the unconscious of society, deeply rooted in people's minds, affecting people's value judgments on both sexes. This makes the report of the elderly, male reports higher than women. Secondly, respecting the old and loving the young is the traditional virtue of our country, especially in Shandong, the hometown of Confucius and Mencius. In today's increasingly aging society, tapping and promoting the traditional culture of respecting the elderly and respecting the elderly is of great significance for caring for the elderly and building a harmonious society. The Qilu Evening News is also related to the traditional culture in shaping and reporting the image of the elderly. In his research, Qi Jiyuan proposed the traditional cultural factors of respecting the elderly and loving the young, which has a reference for the author.

\subsection{Construction of poblic discourse}

Li Yanhong once mentioned that in the process of reflecting objective facts, the media will purposely reconstruct and omit the objective texts. ${ }^{[10]}$ In the selection of news reports, the media staff determines which of the daily events can be reported and which cannot be reported. The subjective awareness of the staff plays an important role in the selection of news events. At the same time, in today's society, the patriarchal culture participates in the mass media and completes the shaping of the female image. This role expectation has developed into the mainstream discourse of the mass media and has become a "discourse power" that has a habitual control over people's daily life and self-awareness. What's more, this discourse is also consistent with the traditional culture of our country, which makes this expectation highly permeable and develops into a gender norm that people generally agree with and abide by in a very short time. In his research, Liu Tao also proposed the important influence of male cultural discourse. The author's analysis is consistent with previous research.

\subsection{Subjective awareness of media practitioners}

The textbooks are constructed by the society, and the thinking structure and subjective interpretation of the editors will influence the presentation of the events. On the one hand, the media practitioners are mostly young people in the city. On the issue of treating the elderly, they have a subjective prejudice against the elderly under the guidance of the preconceived stereotypes. They believe that the elderly are helpless and weak. For young people, the body is not healthy enough, while not paying attention to the necessity of old-age reports, ignoring the existence of rural elderly. This internalized stereotype of the elderly group, whether positive or negative, will affect journalists' reports on the elderly. This negative attitude of the staff directly affects the way they handle the news related to the elderly, making the media image of the elderly appear insufficient, and the reports of the elderly in rural areas are much lower than those reported by the urban elderly. 
On the other hand, for the sake of economic efficiency, the staff will be more inclined to select those eye-catching news reports.

\section{4. the countermeasures and suggestions for the deviation of the image of the elderly}

\subsection{Having an objective view on reporting on the elderly}

Try to reduce the impact of social culture, and treat the reports of the elderly objectively and neutrally. Firstly, change the concept, report the elderly as comprehensively and fairly as possible, and reduce the labeling report. Secondly, use the humanistic perspective as much as possible instead of using the commercialist attitude to report the elderly problem. Finally, pay attention to the positives of the elderly. Upward things, passing positive energy.

\subsection{Building an equal public discourse environment}

At the moment of the male right to speak, the representation of female images is insufficient.If we want to change this situation, on the one hand, we will work hard to eliminate the disadvantages of women in society, enhance women's social and economic status, narrow the gap between men and women in terms of status, thereby changing the social environment of male discourse and creating an equal society surroundings. On the other hand, monitoring the use of the mass media, especially the establishment of the concept of equality of staff and the control of the content of communication, through the training, education and other means to cultivate the equality of staff, from the source of control Content, and strive to spread objective and neutral content of the elderly.

\subsection{Changing the concept of media workers}

Improving media coverage for older people must begin with improving and changing the stereotypes of journalists about older people. Firstly, journalists should be able to introspect and consciously suppress excessive stereotyped thinking and expression in news reporting, establish an unbiased belief strategy, play self-regulation, and improve stereotypes of older groups. Secondly, journalists should be more in contact with the elderly, directly increase their understanding of the elderly, and present a richer image of the elderly in the report. Thirdly, journalists should participate in education, training, and training related to the elderly, and indirectly raise awareness of the elderly. Fourth, set up a special edition for the elderly, enrich the form of reports, and improve the reports of the elderly.

\section{References}

[1] Zhang Ronghua, Research on the Media Image of the Elderly in Huashang Daily, Shaanxi Normal University, 2015.

[2] Liu Bo, The status quo of China's social aging and its countermeasures, Knowledge Economy, vol.17,2013.

[3] National Ageing Office:Research Report on the Trend of China's Population Aging Development, China Women's Movement, vol.2, 2007.

[4] Peng Zengjun, Media Content Analysis Method, Renmin University of China Press, vol.12,2012.

[5] $\mathrm{Bu}$ Wei, On the content analysis method, International Journalism, vol.4, pp.55, 1997.

[6] https://baike.sogou.com/v115614.htm?fromTitle=\%E9\%BD $\% 90 \% \mathrm{E} 9 \% \mathrm{~B} 2 \% 81 \% \mathrm{E} 6 \% 99 \% 97 \mathrm{Qi}$

[7] Du Peng, The current situation and changes of China's population aging, China Social Security, vol.11,2013.

[8] Hu Ling, on the stereotype of reporters' cognition [J], media time, January 2011, P42. 